\title{
Development and Application of Fiber Strain Monitoring System for Ship Structure
}

\author{
Sujun Yang ${ }^{12}$ Huien You $^{3}$ Shihai Hua ${ }^{4}$ Yanqing Wei ${ }^{12}$ \\ ${ }^{1}$ Marine Design \& Research Institute of China, Shanghai 200011 China \\ ${ }^{2}$ Laboratory of Science and Technology on Water Jet Propulsion, Shanghai 200011 China \\ ${ }^{3}$ Veolia Water Solution \& Technologies, Shanghai 200041 China \\ ${ }^{4}$ Wuxi Beetech Sensor Inc., Wuxi 214024 China
}

Keywords: optical fiber strain, ship structure, health monitor system, lab and real ship verifications

\begin{abstract}
Ships are characterized by complex structure and poor operation environment. It is very important to monitor the health state of the ship structure. In the paper, one-way and three-way optical fiber sensors in ship structure monitoring are designed and produced. Related values are simulated. Related samples are developed, loading test is implemented in the laboratory, the precision can meet requirement on real ship monitoring. On the basis, relevant data acquisition device is configured and installed on real ship, and related tests are implemented for proving the reliability of the system.
\end{abstract}

\section{Introduction}

Ship belongs to a large integrated system with complicated structure. It suffers from interaction of various loads when it is navigated in rough sea environment for long term, such as wind load, sea current, wave load, ice load, deep water pressure load, etc. It may be impacted by typhoon, ship collision, explosion, etc. sometimes. The structure still should be affected by environmental corrosion, etc. itself. Therefore, it is particularly important to monitor ship structure stress.

Generally, strain gauge is usually adopted for testing ship structure stress, aging, temperature drift and other effects are generated with the passage of time. Strain gauge has the most common phenomenon of drift. The baseline of output voltage is moved when there is no strain signal. It is called zero drift. Causes of zero drift are roughly divided into two categories: too high bridge circuit voltage and influence of insulation resistance.

Therefore, it is not suitable for long-term monitoring. It is easy to cause reduction of insulation resistance due to moisture of strain gauge. It is easily produced phenomenon in the process of long-term monitoring. The phenomenon is easily produced during and after patching, and component grinding and strain gauge protection should be specially emphasized. However, fiber strain can avoid the above defects compared with normal strain gauge. On the basis, new sensors are developed on the basis of fully considering current optical fiber sensors in China and foreign countries. Related tests were implemented.

\section{System design idea}

Fiber strain monitoring system for ships is mainly used for changing to-be-measured physical quantities into fiber grating wavelength through fiber grating strain sensors. The following physical quantities are mainly monitored: stress, bending moment and torsion deformation, etc. on key parts of the ship. 'Ship deformation monitoring' functions are realized. The design ideas are shown in figure 1. 


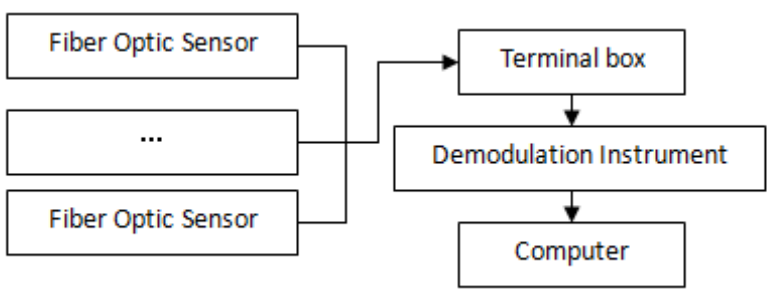

Fig.1 Design idea of fiber strain monitoring system

\section{Design principle of fiber grating sensing}

\subsection{Principle of fiber grating sensing}

Fiber grating refers that ultraviolet lithography is used for writing reflection grating into silicon depositing single-mode fiber (single grating), or short laser pulse interference patterns are used for writing reflection grating into silicon depositing single-mode fiber (grating array) in the process of fiber annealing more directly and more effectively. Fiber grating can reflect a section of narrow-band light of narrow spectrum with Bragg as wavelength and $\lambda \mathrm{B}$ as center aiming at light transmitted in the fiber core. After broadband light is transmitted into the grating, the light with wavelength of $\lambda B$-reflection wavelength in the center of grating is reflected back. The light with other wavelengths penetrate through the grating. Fiber grating reflection spectrum has spectral width $<0.3 \mathrm{~nm}$. Because grating has such characteristics, many grating sensors can be connected on one piece of optical fiber in series.

\section{2 fiber grating demodulation principle}

Tunable F-P cavity demodulation method is used in the system. Tunable F-P (Fabry-Perot) filter is a kind of optical device for demodulating fiber grating, wherein F-P cavity interference is utilized for producing optical filter function.

F-P cavity selectively outputs light in wavelength with extremely high interference under the effect of piezoelectric ceramic, thereby playing the role of tunable harmonic wave. The incident light enters into the F-P cavity composed of two parallel mirrors. Phase difference is produced between two adjacent light rays.

$$
\delta=\frac{4 \pi n h \cos i}{\lambda}
$$

In the formula, $\mathrm{n}$ refers to refractive index, h refers to the distance between two mirrors, $\mathrm{i}$ refers to angle of incidence, and $\lambda$ refers to wavelength.

Light with some specific wave lengths, which meet related conditions, can interfere D within certain spectral range due to interference of multiple beams, and extremely high interference can be produced, and $\lambda \mathrm{k}$ should meet the following conditions when $\mathrm{i}=0$ (parallel light with incident angle of $0)$ :

$$
2 \mathrm{nh}=\mathrm{K} \lambda \mathrm{k}
$$

In the formula, k refers to interference coefficient, and light with some specific wavelengths can penetrate through F-P cavity. One of two high reflector mirrors in tunable F-P cavity is fixed. The other reflector mirror can be moved, and piezoelectric ceramic is attached on the back. Different voltages are applied on the piezoelectric ceramic; thereby promoting it to produce different pressures, and the mobile high reflector mirror can be moved.

Therefore, cavity length H of F-P cavity is changed, and interference conditions can be changed, therefore the light wave penetrating through F-P cavity also can be changed, thereby realizing the tunable purpose of transmitted light wavelength $\lambda \mathrm{k}$.

\section{Structure design of fiber grating sensing device structure}

Fiber grating should be specially packaged to realize the following purposes aiming at sensors applied for ship structure monitoring: 
Protection: since bare fiber grating is relatively weak, it is prone to damage by external forces; peripheral protection must be used for avoiding sensor damage.

Compensation: because the fiber grating is sensitive to stress temperature, the temperature sensitivity should be reduced as far as possible during stress measurement.

Capacity expansion: optical fiber allowable strain is $3000 \mu \varepsilon$, the range can not meet use requirement in actual application occasions, and certain mode must be used for increasing the range.

In the paper, the following encapsulation structure is designed as shown in figure 2 based on the above purposes. Sensor encapsulation structures are divided into side A and side B, which are respectively used for feeling extrusion pressure and tension. Side A includes two cantilevers. When components suffer from extrusion pressure, components are compressed, two cantilevers are moved relatively, the distance between two fixed points is increased, grating A is stretched, and grating B is in the Free State. Side B fixed points are moved to reverse direction when the components are stretched, the grating B is stretched, and grating B is in free stage. Grating A and grating B are in the free stage during encapsulation.

Grating $\mathrm{A}$ wavelength offset is set as $\Delta \lambda \mathrm{A}$. Grating $\mathrm{B}$ is set as $\Delta \lambda \mathrm{B}$. Overall strain of the component $\Delta \lambda$ has the following relationships: $\Delta \lambda=\Delta \lambda \mathrm{B}-\Delta \lambda \mathrm{A}$.

Since the same specification is selected by grating $A$ and grating $B$, wavelength offset counteraction can be caused by temperature after reduction, the system range is improved as twice of the original value. When the gratings suffer from extrusion pressure, the different value result is negative. When the gratings suffer from tension, the difference value result is positive.
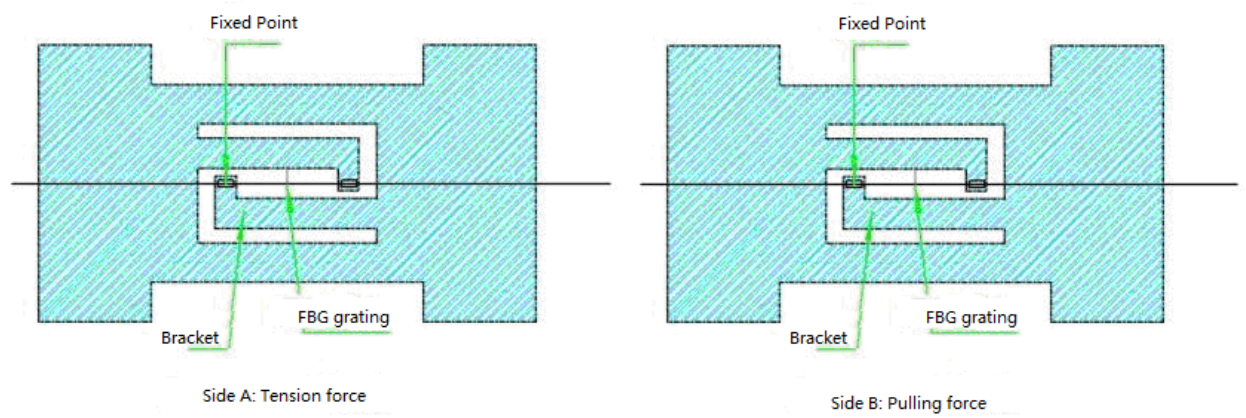

Fig.2 Encapsulation of fiber grating sensor

\section{One-way sensor and test}

\subsection{Design}

In the paper, sensors with the following form structure are designed based on the principle of optical fiber sensor (shown in figure 3). They are made of mold stainless steel. Automatic difference compensating mode is adopted as measurement mode. The range is 5000 strains.

\subsection{Test and verification ideas}

In the design process, initial structure size is determined for design firstly through theoretical calculation. Finite element is analyzed after design. The design is adjusted according to design results until design requirements are reached. After finite element analysis meets design requirements, samples are processed, optical fiber is packaged, and the sensor is fixed on one ship steel plate after packaging. Actual use condition is stimulated for calibration and verification on the tensile machine.

Figure 4 shows that when the structure is simulated, the displacement of the measured zone is about 1.8 times of the fiber grating fixed zone. Namely, when $5000 \mu \varepsilon$ is produced on the lower portion, the fiber grating displacement is smaller than $3000 \mu \varepsilon$, which is within the elastic strain scope of optical fiber. 

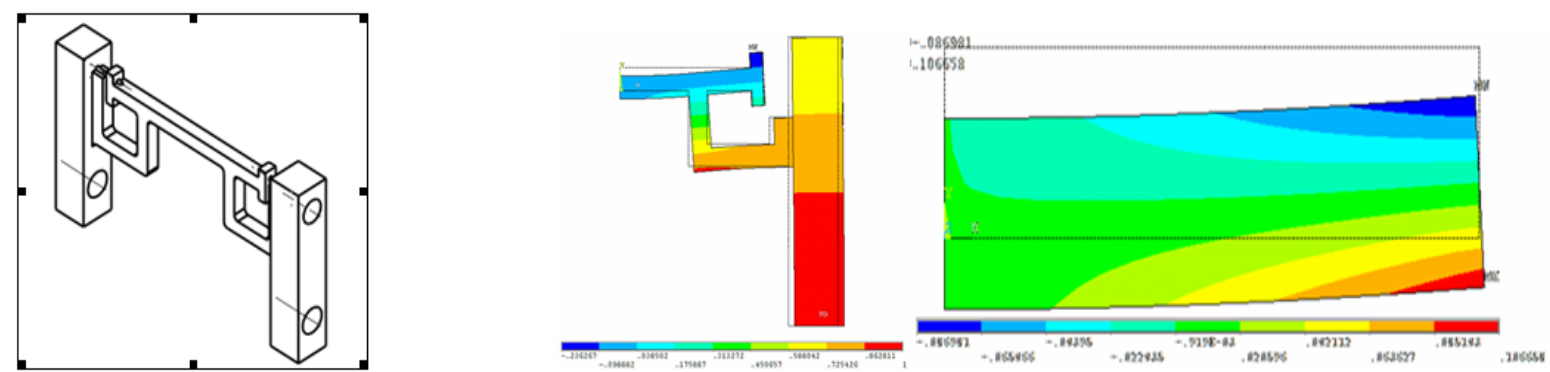

Fig.3 One-way sensor schematic diagram Fig.4 One-way sensor finite element analysis diagram

\subsection{Test and verification result}

After finite element analysis meets the requirements, the product is processed according to drawings. It is calibrated and verified on standard tensile machine after encapsulation. The result data is analyzed.

To-be-measured component interval is from zero to $600 \mathrm{kN}$, strength experiment with interval of $100 \mathrm{kN}$ is implemented. Each force full load is stably maintained for 5 minutes. Fiber grating readings and strain gauge readings below the sensor are collected synchronously. The following results are obtained after analysis as shown in Table 1 . Table 1 shows that the maximum deviation rate is $0.441 \%$, which can satisfy the requirement of real ship test.

Table 1 The calculation and measurement result of one-way sensor

\begin{tabular}{|ccccc|}
\hline Load & Calculation & Measurement & Deviation & Deviation ratio \\
$\mathrm{kN}$ & $\mu \varepsilon$ & $\mu \varepsilon$ & $\mu \varepsilon$ & $\%$ \\
100 & 595.445 & 597.383 & 1.938 & $0.048 \%$ \\
200 & 1153.808 & 1156.7 & 2.893 & $0.072 \%$ \\
300 & 1690.273 & 1689.691 & 0.583 & $0.015 \%$ \\
400 & 2207.668 & 2209.179 & 1.511 & $0.038 \%$ \\
500 & 2715.174 & 2717.541 & 2.367 & $0.059 \%$ \\
600 & 3218.795 & 3201.166 & 17.628 & $0.441 \%$ \\
\hline
\end{tabular}

\section{Three-way sensor}

\subsection{Optical fiber sensor design}

Three modified one-way sensors are adopted in the three-way sensor, which are arranged according to 45 degrees in turn around one public point. The layout and structure appearance are shown in Figure 5. In the paper, sensors in the following form structure are designed based on the principle of optical fiber sensor. The sensors are made of mold stainless steel. Automatic difference compensating mode is adopted as measurement mode. The range includes 4000 strains. The fiber grating region is closer to the measured surface for plane strain. The measurement results are better. In addition, the anti-interference ability is better; the single arm components for three-way sensor are optimized for treatment.

\subsection{Test and verification ideas}

The interference of shear force on sensor can be minimized through adjusting the thickness proportion of fiber grating paste surface and adjacent surface. The measurement range of the sensor can reach $\pm 4000 \mu \varepsilon$. Finite element analysis is carried out on the design (please see figure 6). The displacement of the to-be-measured area is about 1.5 times of the fixed zone of fiber grating, namely when $4000 \mu \varepsilon$ is produced on the lower portion, the displacement of fiber grating is smaller than $2700 \mu \varepsilon$, which is within the elasticity strain scope of optical fiber. 


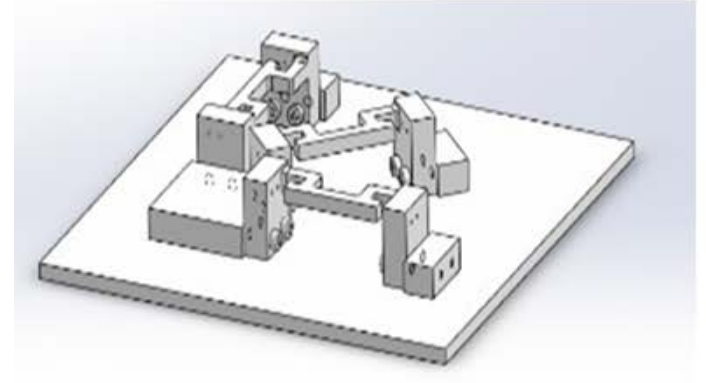

Fig.5 Three-way sensor schematic diagram

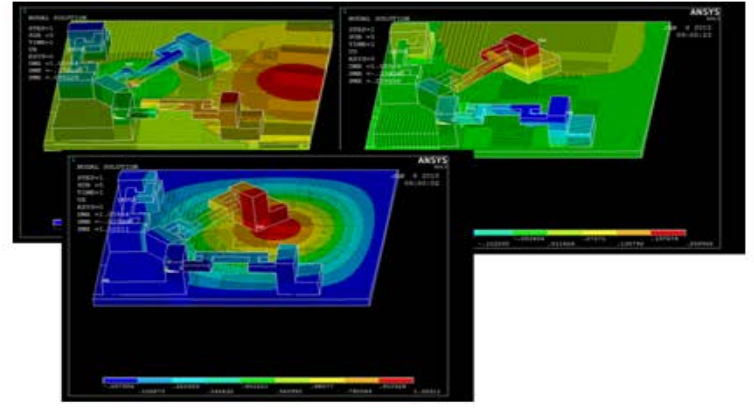

Fig.6 Finite element analysis of three--way sensor

\subsection{Test and verification result}

After the finite element analysis meets the requirements, the product is processed according to drawings. It is calibrated and verified on standard tensile machine after encapsulation. The result data is analyzed. Figure 6 shows that to-be-measured component interval is from zero to $600 \mathrm{kN}$, each force full load is stably maintained for 5 minutes at interval of $100 \mathrm{kN}$. Fiber grating readings and strain gauge readings below the sensor are collected synchronously. The following results are obtained after analysis as shown in Table 2.

Table 2 The calculation and measurement result of three-way sensor

\begin{tabular}{|ccccc|}
\hline Load & Calculation & Measurement & Deviation & Deviation ratio \\
kN & $\mu \varepsilon$ & $\mu \varepsilon$ & $\mu \varepsilon$ & $\%$ \\
100 & 705.971 & 667.480 & 38.491 & 0.481 \\
200 & 1296.327 & 1314.677 & -18.350 & 0.229 \\
300 & 1852.183 & 1835.676 & 16.507 & 0.206 \\
400 & 2387.622 & 2423.773 & -36.151 & 0.452 \\
500 & 2918.836 & 2911.719 & 7.117 & 0.089 \\
600 & 3444.770 & 3434.801 & 9.969 & 0.125 \\
\hline
\end{tabular}

Force of $0,100,200,300,400,500$ and $600 \mathrm{kN}$ is loaded on measuring parts of welding sensor in order to verify the three-way relationship as shown in figure 10. In figure 7, series 3 belongs to tensile direction, series 2 belongs to $90^{\circ}$ direction, and series 1 belongs to $45^{\circ}$ direction. Y direction can be shortened and negative strain can be produced during tension at $\mathrm{X}$ direction as a result of the existence of Poisson's ratio. Strain at $45^{\circ}$ direction is regarded as vector synthesis of strain at axis $\mathrm{X}$ and axis Y, which is basically consistent with theoretical calculation.

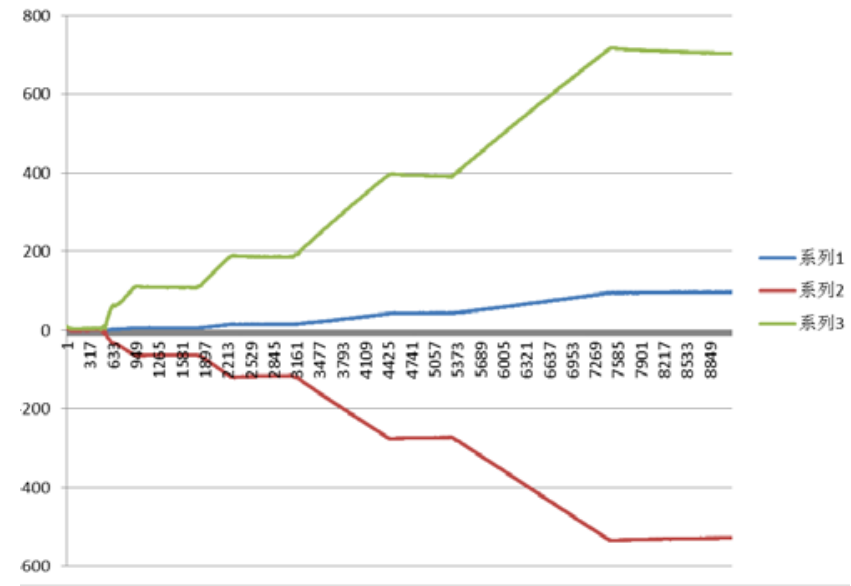

Fig.7 Validation of three-way sensor 


\section{System installation and validation}

\subsection{Arrangement of measuring points}

If one monitoring point is only affected by one-way compressive stress, one strain sensor can be arranged along the load direction, if load is complex on the monitoring point, three-way strain sensor should be arranged.

The product is installed on cross section girder or end portion 1/4L, 1/2L and 3/4L (L refers to ship length) to the stem, which is mainly used for measuring total longitudinal strength bending moment of the hull;

The product is installed on broadside reinforcement plate, which is used for measuring the stress role of seawater pressure on broadside;

The product is installed on transverse bulkhead, which is used for internal stress of bulkhead, etc.;

The product is installed on local open part, which is used for measuring the damage due to local stress concentration, etc.;

The product is installed on the bottom plate of the ship, which is used for measuring total longitudinal bending moment thereof;

\subsection{Test results}

The sensor developed in the paper is installed on one real ship for preliminary experiment. Figure 8 shows stress change state of strain monitoring points arranged in the ship. The figure shows that the stress change value scope of the monitoring point within 72 hours is between 88.12 and $-87.60 \mu \varepsilon$.

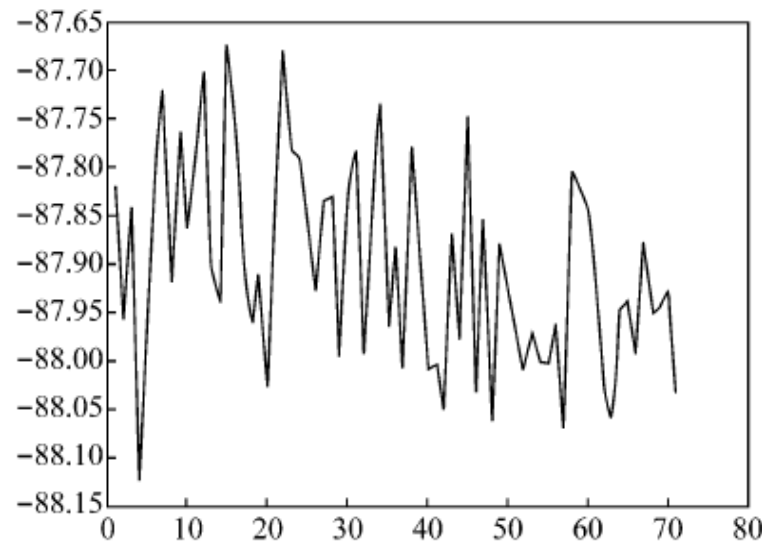

Fig.8 Strain state parameters in the mid-ship area

\section{Summary}

In the paper, one-way and three-way optical fiber strain sensors for real ship monitoring are designed based on the principle of optical fiber sensor. Related lab and real ship verifications are implemented. It can meet requirements on real ship monitoring according to verification. The system development can provide a lot of effective data for long-term monitoring of ship structure. Structure defects can be discovered in advance, and it has excellent guidance effect on safe navigation of the ship.

\section{References}

[1] Zheng Buxiang, Song Yonglun, Zhang Dongsheng, Jiang Desheng. Application of fiber grating sensor in welding stress online monitoring [J]. Welding. 2008 (7):37-42.

[2] Zhu Yunxiang, Mao Ruquan, Fan Jingfeng. Application of fiber grating stress monitoring technology on ships [J]. Transportation Science and Technology, 2013 (3):165-168.

[3] China fiber sensor market trend and investment value study report from 2012 to 2016.

[4] In-depth analysis on fiber grating sensor industry from 2016 to 2021 and guidance report of the '13th five-year' development plan. 